\title{
Developing a four-tilted-mirror telescope as a student project
}

G. Fütterer, A. Sperl, S. Killinger, A. Engelbrecht, M. Werni, et al.

G. Fütterer, A. Sperl, S. Killinger, A. Engelbrecht, M. Werni, W. Krais, "Developing a four-tilted-mirror telescope as a student project," Proc. SPIE 10741, Optics Education and Outreach V, 1074100 (14 September 2018); doi: $10.1117 / 12.2320542$

SPIE Event: SPIE Optical Engineering + Applications, 2018, San Diego, California, United States 


\title{
Developing a four-tilted-mirror telescope as a student project
}

\author{
G. Fütterer, A. Sperl, S. Killinger, A. Engelbrecht, M. Werni, W. Krais, \\ Deggendorf Institute of Technology, Dieter-Görlitz-Platz 1, 94469 Deggendorf, Germany
}

\begin{abstract}
The Faculty of Applied Natural Sciences and Industrial engineering, which is a part of the Deggendorf Institute of Technology (DIT), transfers a broad spectrum of knowledge to the students. Edifying the interrelations, which are present between seemingly isolated fields of knowledge, is a permanent process. In order to make this practical, a telescope construction project was launched.

The Technology Campus Teisnach bundles capacities for process development, production and measurement of highprecision optics. This also includes telescope optics. This qualifies the Campus for being the base of the in-house project.

Fixed boundary conditions are e.g. $400 \mathrm{~mm}$ diameter of the primary mirror M1 and the objective to realize an image performance, which is equivalent to commercial telescopes. Furthermore, an unobscured tilted-mirror-system should be realized. The optical design, which was chosen as a result of an analysis of the state of the art, is based on a subset of the parameter space, which was published in 1989 by M. Brunn ${ }^{1,2}$. The concept was later built by D. Stevick as f/12-system (with reference to the work of M. Paul, 1935) ${ }^{3}$.

The DIT project started with a comparison of $\mathrm{f} / 7$-systems. They had been implemented in the optical design software Zemax. The imaging performance was compared within a field of view of $0.7^{\circ} \mathrm{deg}$. The mechanical design includes FEM simulation of thermal effects on slightly weighted mirrors. Different tubes had been compared, including carbonfiber-reinforced-polymer (CFRP) Monocoquetubus. Another task is the realization of fast and precise tracking. The state of the development is set out.
\end{abstract}

Keywords: Unobscured four-tilted-mirror telescope, wave front based alignment, CFRP monocoque tube, FEM simulation, student project.

\section{INTRODUCTION}

The Deggendorf Institute of Technology (DIT) is equipped with state-of-the-art facilities and excellent technological support. The educational tasks cannot always be clearly separated between the individual faculties. But the cooperation is very good between them. A broad range of knowledge is taught at the Faculty of Applied Natural Sciences and Industrial Engineering $(\mathrm{NuW})$, which is part of the DIT in Bavaria, Germany too.

The question may arise why to choose a technological complex setup like a 4-tilted-mirror telescope as a student project. One educational goal is to prepare the students optimally for their working live. However, the changes are fast and they will become even faster, especially in technological areas. Furthermore, the complexity and the diversification will increase too. And in addition, more and more activities and processes will be automated and/or digitalized. As a consequence, to rely on isolated, fixed and so called crystalline knowledge alone cannot be the solution to this challenge. A focus has to be on the development of problem analysis skills. Due to the increasing complexity of technical systems, this has to be done within a thematically diversified development team. In order to make this more practical, a telescope construction project was launched. In other words, it was found that the development of a 4-tilted-mirror telescope is reasonable complex and contains enough interdisciplinary aspects, which can be developed within a project, which is based on mutually synchronized team work. One point is that isolated knowledge and isolated solutions do not work here. For all working packages, the boundary conditions of the final system have to be taken into account. Thus, knowledge transfer and barrier free discussion within the teams is a must have. And this works fine.

At the beginning, which was 2016 already, there was a decision made at an internal DIT meeting. A student project should be started. All interested students should be able to take part. The target should be reasonable complex and impressive enough in order to generate attraction to internal and external students. An unobscured mirror telescope was

Optics Education and Outreach V, edited by G. Groot Gregory, Proc. of SPIE Vol. 10741, 1074100

(C) 2018 SPIE · CCC code: 0277-786X/18/\$18 · doi: 10.1117/12.2320542 
identified as a potential target. One boundary condition was that it is not a copy of a telescope, which is already commercial available, scaled or not. Another boundary condition was the diameter of the largest mirror used, which should be at least within the range of $400 \mathrm{~mm}$ to $500 \mathrm{~mm}$. The optical resolution should be within the range that extends from amateur telescopes to professional telescopes. This was the starting point for the study of existing optical designs and existing mounting options too.

An important factor for technology projects as e.g. the one, which will be discussed herein, are the in-house capabilities of the DIT. Several external units, which are called technology campus, act as development and technology transfer center. One of them is the Technology Campus in Teisnach. This technology campus is the DIT's basis for the production and measurement of high-precision refractive optics and reflective telescope optics too. In other words, the DIT can make and measure the optical components. This also holds for most of the mechanical and electronical parts. Thus, the students have the opportunity to understand optics, mechanics, sensors and automatic control engineering within their complex interplay.

\section{MECHANICAL DESIGN OF THE TARGET APPLICATION}

\section{Telescope tube design}

In this section, a generic overview on the mechanical design of the telescope tube will be given. With regards to the material selection, there are several possibilities. For instance, a classic mechanical setup can be implemented by using plates and tubes, which are made of standard metal alloys as e.g. EN AW 7075 (AlZn5,5MgCu), FeNi36, Fe-33Ni-4.5Co or Ti6Al/4V. However, the different alloys are related to different pros and cons. The use of aluminum alloys can provide a lightweight and most cost efficient solution. The drawback is the large coefficient of thermal expansion of e.g. $23.410^{-6} / \mathrm{K}$. Although Fe-33Ni-4.5Co has a very low coefficient of thermal expansion of $0.5510^{-6} / \mathrm{K}$ only, it is much more expensive and much denser than aluminum alloys. The coefficient of thermal expansion of $\mathrm{Ti} 6 \mathrm{Al} / 4 \mathrm{~V}$ is $8.710^{-6} / \mathrm{K}$ and thus close to the one of a plurality of optical glasses. This makes Ti6Al/4V an interesting material for mounting refractive optics working in transmission. However, the primary mirror M1 is made of the glass ceramic Zerodur. Zerodur has a coefficient of thermal expansion of down to $0 \pm 0.00210^{-6} / \mathrm{K}$ (expansion class 0 ). This points out that there is no clear metal alloy preference for the telescope tube design. And this is the reason why carbon-fiber-reinforcedpolymer (CFRP) might be used to fill the parametric gap between, weight, coefficient of thermal expansion and costs.

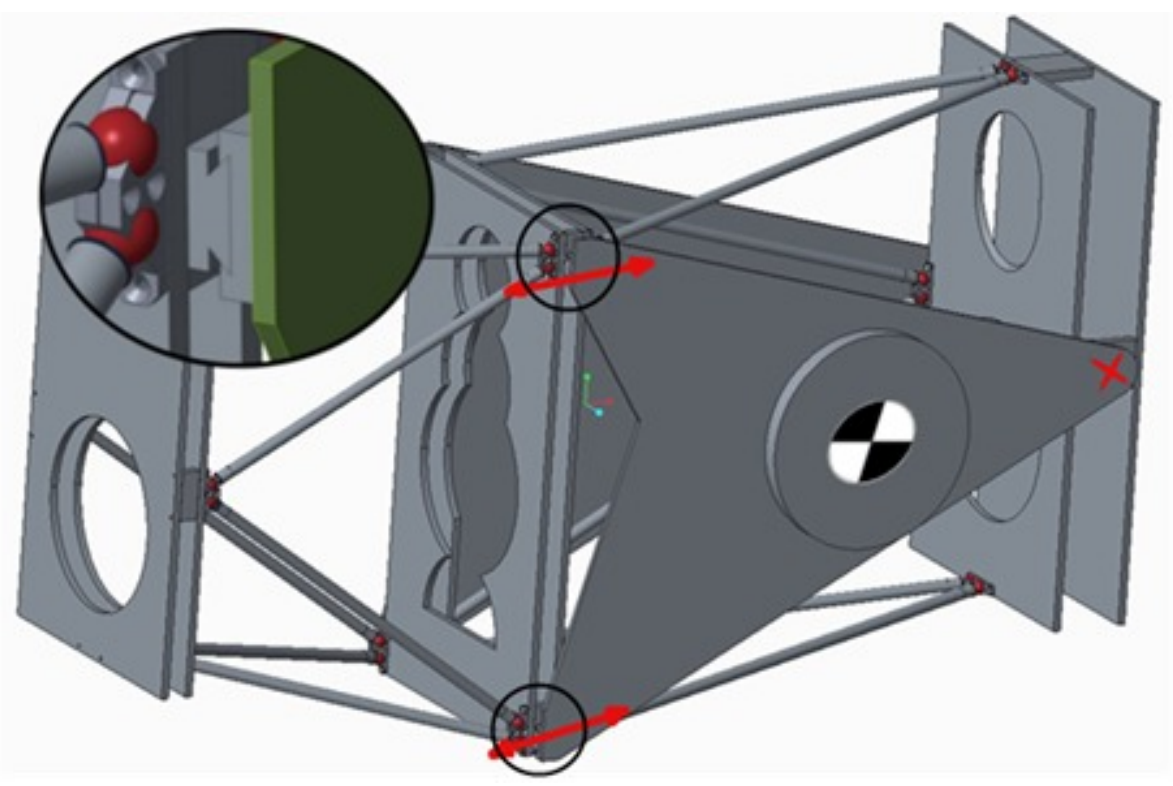

Figure 1. Pseudo 3D view of a telescope tube, which is based on carbon-fiber-reinforced-polymer (CFRP) tubes and plates. Originally this was designed for an $\mathrm{f} / 7$ system. The mounting axis, which provides the connection to the parallactic mounting fork, goes through the center of gravity. 
Figure 1 depicts a pseudo 3D view of a telescope tube, which is based on plates and tubes. The material of the plates and tubes is carbon-fiber-reinforced-polymer (CFRP). The original mechanical design was scaled in order to fit an f/7 telescope system. The entrance has a diameter of approximately $410 \mathrm{~mm}$ and is placed at the lower left hand side of the figure. The mechanical design shown make use of standard components. That keeps the costs down. Using CFRP is related to several boundary conditions. The elasticity modulus is not isotropic. The coefficient of thermal expansion depends on the discrete mesh and is not isotropic. The preferred material, which is commercial available for a reasonable low price, is $3 \mathrm{~K}$ plain weave. The use of CFRP is explicit a preferable constructive option and not related to design aspects as the styling, which might be also important of course.

An alternative approach is to use a monocoque as a telescope tube. A CFRP monocoque tube is shown at the right hand side of Figure 2. The CFRP structure is tightly adjusted to the optical path of the 4-tilted-mirror-telescope. The construction, which leaves the path of classic telescope tube design, is compact, lightweight and can provide the required stiffness. An expanded polystyrene foam core, which is shaped like the optical path, can act as the preliminary core to be wrapped. A solvent can be used to get the foam finally out of the monocoque tube structure.

\section{Parallactic mounting}

The target telescope will be designed for semi-fixed usage. The DIT in Deggendorf and the Technology Campus in Teisnach are the two planned places of installation. This is the reason why a parallactic mounting will be used, which has a fixed parallactic angle. The angular difference, which will be present between Deggendorf and Teisnach, can be adjusted easily within the base unit. The parallactic mount is depicted at the left hand side of Figure 2.
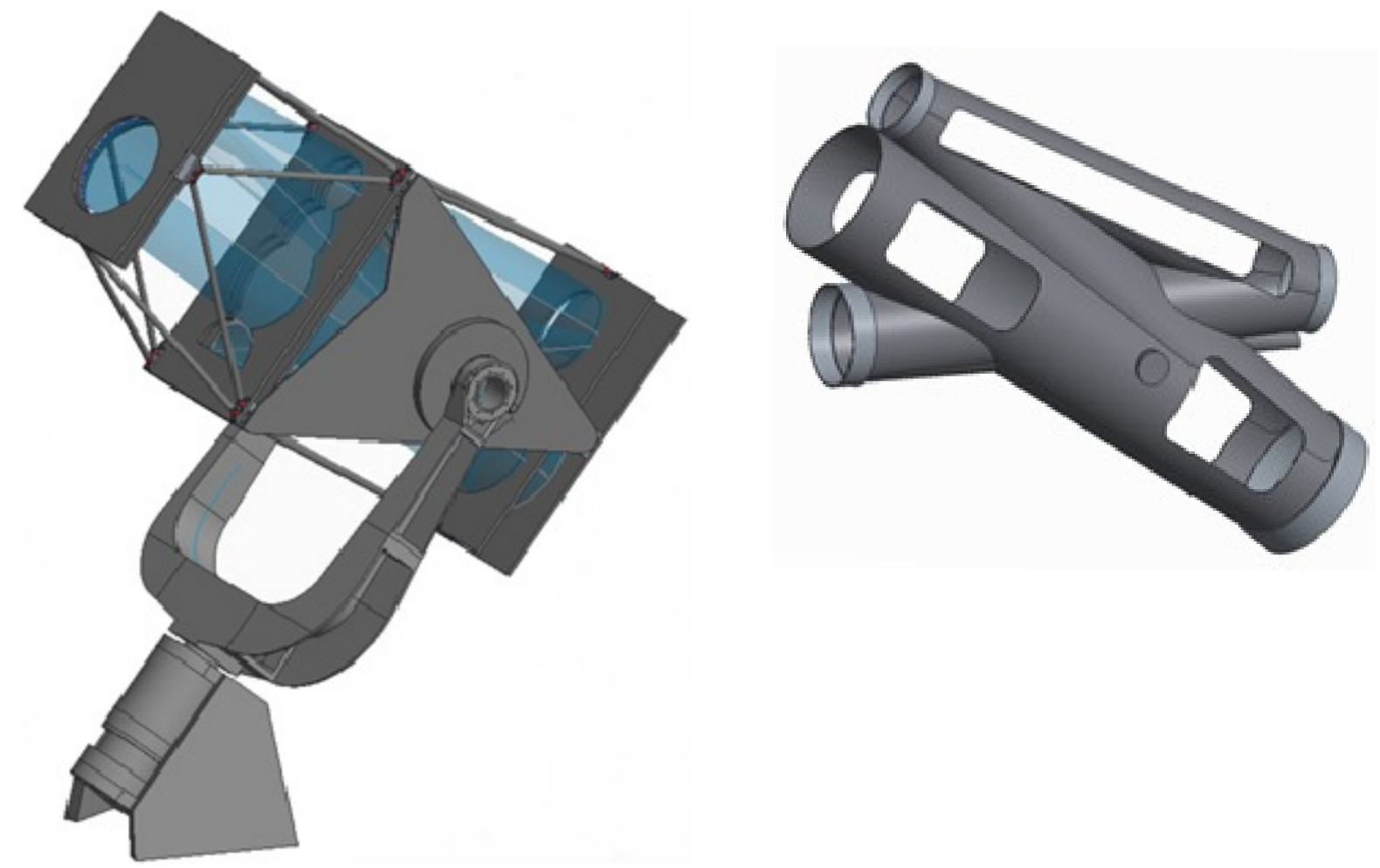

Figure 2. Left: Pseudo 3D view of a telescope tube in a parallactic mounting. Right: Pseudo 3D view onto a CFRP based monocoque telescope tube. The light gray metal rings indicate the compact mountings of the mirrors M1 to M4. The light enters the systems from the left hand side.

The length of the tubes, which are shown in Figure 2, is approximately $1.6 \mathrm{~m}$. Parallactic fork mount provides a simple control. The rotation of the earth is compensated by using one axis only. In contradiction to the azimuth mounting, it is easy to avoid image distortion due to this corrective movement. The tube is supported on two sides. This enables a reasonable high stiffness and a lightweight construction of the entire embodiment. The fork reminds of a motorcycles 
swing arm, which is related to the function to be realized. The fork is made of five main segments. This can be seen at the left hand side of Figure 2. The segmentation reduces the manufacturing cost significantly.

A boundary condition is to catch the ISS and to follow the space station with an uncertainty of equal or less than one detector pixel, e.g. for a few minutes. Otherwise reasonable sharp images would not be obtained. Absolute angular encoder can be used in order to provide the required angular resolution. For instance, the absolute encoder RCN 8510 from Heidenhain provides a system accuracy of \pm 1 ”, which is $\pm 1 / 3600^{\circ} \mathrm{deg}$. For the RCN 8510 the position error per signal period is \pm 0.15 ". The hollow through shaft with a diameter of $100 \mathrm{~mm}$ provides design freedom in regards to the cable feedthrough. Torque direct drives provide high dynamics. And if combined with a sufficient encoder they can also provide the angular accuracy, which is required for this telescope. In other words, the combination of an angular encoder and a torque direct drive provides best controllability. In addition, the transmission losses can be neglected. Cross roller bearings enable the highest accuracy of roller bearings. They are also used in measurement applications.

\section{Mirror mounting}

The diameter of the primary mirror M1 is $400 \mathrm{~mm}$. The Thickness of the mirror M1 is $50 \mathrm{~mm}$. The mirror mount, which was designed for the primary M1, is depicted at the right hand side of Figure 3 by using a pseudo 3D view. 18 contact pads had been implemented. The arrangement enables gimbal operation, which means that the center of rotation of the two axis is at the point $\mathrm{P}(0,0,0)$ of the parabola defining the mirror surface.
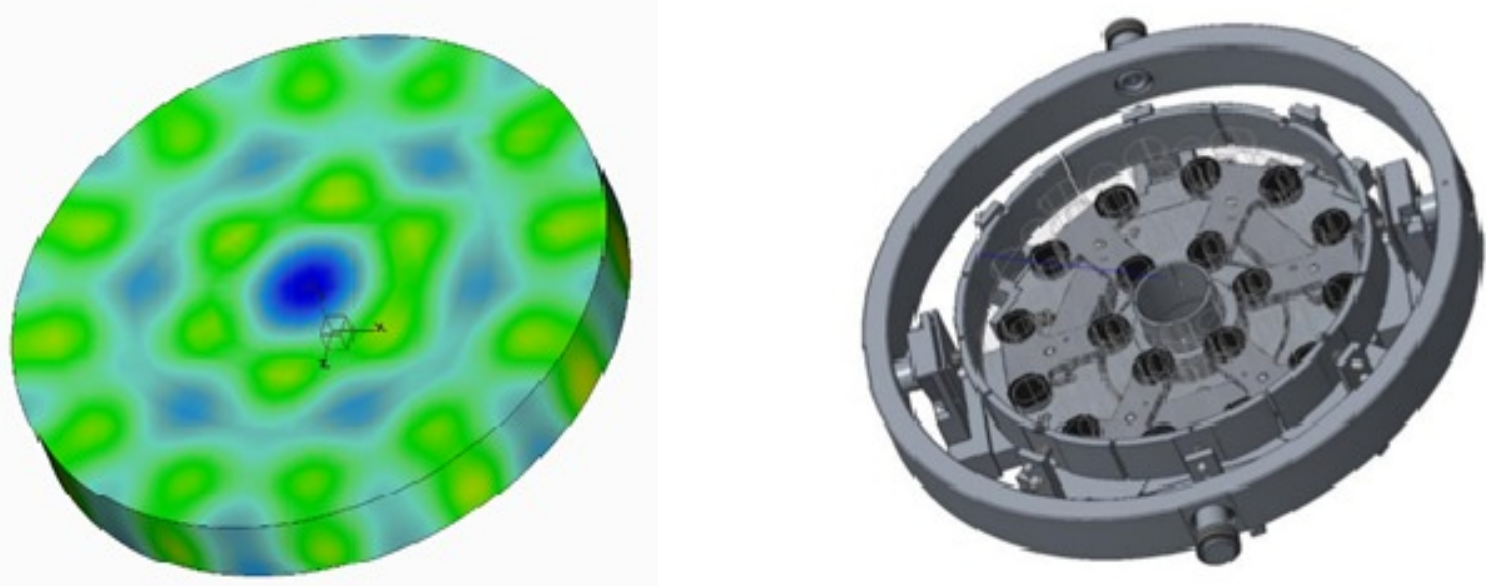

Figure 3. Left: Pseudo 3D view of a FEM simulation of the mounted primary mirror M1. Right: Gimbal mount, which was used within the simulation. The number of contact pads is 18 . This is sufficient for a mirror diameter of $\mathrm{d}_{\mathrm{M} 1}=400 \mathrm{~mm}$.

The finite elements method (FEM) simulations of the M1-position change has shown that the related change of the surface figure is in the range below $10 \mathrm{~nm}$. This low form error was obtained by using the gimbal mirror mount, which is depicted at the right hand side of Figure 3. Presuming a wavelength of $\lambda=550 \mathrm{~nm}$, this is related to a reflective wave front error of approximately $\Delta \varphi(\mathrm{PV}) \cong \lambda / 30$. One intermediate conclusion is that 18 contact pads are sufficient for the M1 mirror mount. However, the gimbal mount, which is shown in Figure 3, is still too bulky and too heavy. One point is that this mirror mount provides much more angular tilt range than required. In regards to manufacturing and alignment tolerances an angular range of less than $\pm 0.5^{\circ} \mathrm{deg}$ is sufficient. An alternative to the mechanical construction, which is shown in Figure 3, a matching pair of two small spherical ring segments can be used in order to provide the gimbal operation. The 18 contact pads, which are arranged in six tiltable groups, still can be used. In other words, the mechanical construction can be shrunk significantly. This reduces the volume, increases the stiffness and reduces the weight. One of the next steps is to realize a lightweight structure of the M1 backside. This can be done with the ultrasonic CNC machining center US40 from DMG Mori, which can handle the $400 \mathrm{~mm}$ M1. This is part of the DIT capabilities.

The light gray metal rings, which can be seen at the right hand side of Figure 2, indicate the compact gimbal mirror mountings of the mirrors M1 to M4. The mechanical design of these mirror mounts is ongoing and not finished yet. 


\section{OPTICAL DESIGN OF THE 4-TILTED-MIRROR-TELESCOPE}

\section{Telescope design}

In this section, a generic overview on the optical design of the telescope will be given. The optical design is based on a subset of the parameter space, which was published by M. Brunn in 1989 already (DE3943258C2) ${ }^{1,2}$. A similar concept was later built by D. Stevick as f/12 system (with reference to the work of M. Paul, 1935) ${ }^{3}$. The DIT student project started with a comparison of $\mathrm{f} / 7$ systems, which had been implemented in the optical ray-trace software Zemax (among others Newton, Cassegrain, Ritchey-Chrétien). The imaging performance was compared to a field of about $0.7^{\circ}$ deg in total.

The optical path of the 4-tilted-mirror-telescope is depicted in Figure 4. The light enters the telescope from the left (see white arrow).

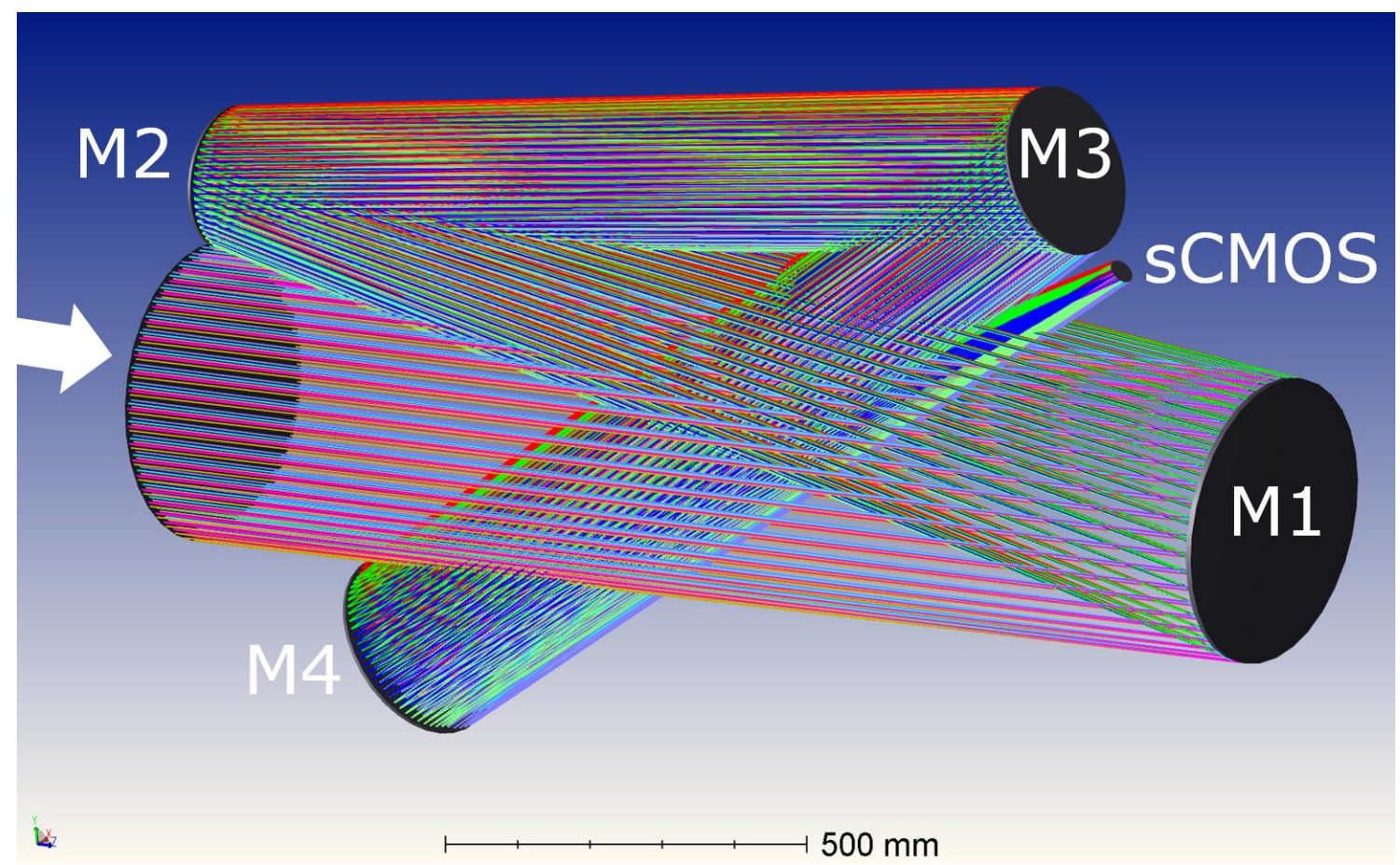

Figure 4. Optical path of the $\mathrm{f} / 8$ telescope $(\varnothing \mathrm{M} 1=400 \mathrm{~mm}, \mathrm{tM} 1=50 \mathrm{~mm}$, material: Zerodur, M1: parabolic mirror, M2: convex spherical mirror, M3: plane mirror, M4: concave spherical mirror. The entrance pupil of the system is placed at the left hand side.

The primary mirror M1 is a parabolic mirror, which is slightly tilted. The secondary mirror M2 is a convex spherical mirror, which collimates the convergent light, which propagates behind the primary mirror M1. The secondary mirror M2 is placed within the focal length of the primary mirror M1. Despite the fact that this is a telescope, which make use of tilted mirrors, this aspect reminds on a Galilei telescope. The third (tertiary) mirror M3 is a plane mirror, which folds the optical path. This makes the telescope slightly more compact. The fourth mirror M4 is a concave spherical mirror with $|\mathrm{R} 4|=|\mathrm{R} 2|$. The angle, which is present between the detector plane and the optical axis, is slightly of $90^{\circ} \mathrm{deg}$. A so called full format detector size was presumed. The aim is to have at least 3 pixels for the smallest dissolved spatial period $^{4}$.

Up to this point simple mirror geometries are used, which can be tested in reasonable simple measurement arrangements. Up to now no aspherization is used. However, this might be done e.g. for a second or even third version of this telescope.

\section{Spot diagram and PSF}


The light of a single star is represented by a plane wave entering the telescope with a defined solid angle, which can be split in a defined azimuthal and horizontal part. The captured angular range is given by the field of view (FOV). A representative spot diagram of the $\mathrm{f} / 8$ telescope is depicted at the left hand side of Figure 5 . The field of view (FOV) is $\pm 0.31^{\circ} \mathrm{deg}$. The question may arise, why is the on-axis point spread function (PSF) that worse? The tilt and the z-shift of the detector plane was chosen in a way to minimize the detector planes integral Strehl ratio. An optimization of the on-axis PSF decrease the integral value.
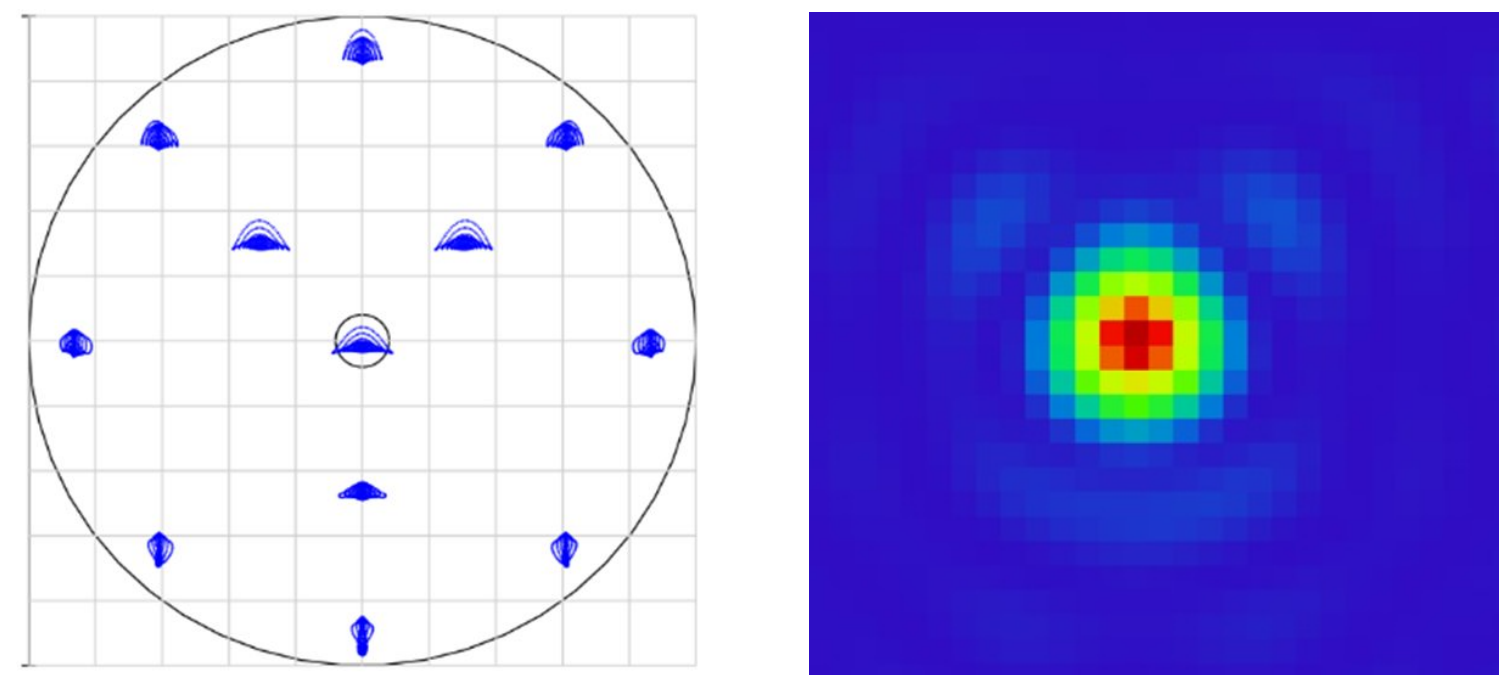

Figure 5. Left: Foci with a magnification of $300 \times$ at a $36 \mathrm{~mm}$ x $36 \mathrm{~mm}$ detector plane. The radius of the systems Airy distribution is r01Airy $=5.4 \mu \mathrm{m}$. Right: intensity distribution of a PSF $\measuredangle$ in at $(-0.31,0){ }^{\circ} \mathrm{deg}$ with diffraction effects, $\lambda=550 \mathrm{~nm}$. The Strehl ratio of the point spread functions is $\geq 0.8$. Thus, the $\mathrm{f} / 8$ telescope is diffraction limited.

In the center of the spot diagram, which can be seen at the left hand side of Figure 5, a black ring can be seen. The diameter is equivalent to $2 \times$ r01Airy $=2 \times 5.4 \mu \mathrm{m}=10.8 \mu \mathrm{m}$. This central part of a PSF is used for the calculation of the Strehl ratio (SR). However, the rays used for the ray tracing are only an approximation. Wave fronts are sampled and the segments local wave front normal vectors are interpreted as rays. But there are no physical rays. Ray tracing is fast and spot diagrams are practical in order to get information on the systems imaging performance. The twelve spots, which are shown at the left hand side of Figure 5, represent twelve plane waves, which are focused onto the detector plane. In order to prove the evidence of the spots diagrams information the diffractive PSF can be calculated. At the right hand side of Figure 5 the intensity distribution of a PSF is shown, which takes diffraction into account. The field angle of this PSF is $\measuredangle$ in $=(-31,0)^{\circ}$ deg. The wave length use for the calculation is $\lambda=550 \mathrm{~nm}$. The telescope has a low numerical aperture (NA). There is a good agreement between the spot diagram based PSF and the diffractive PSF. The change from a f/7 telescope to a $\mathrm{f} / 8$ telescope enables diffraction limited imaging over the entire FOV.

\section{ALIGNMENT STRATEGY}

The alignment of tilted mirror telescopes is much more complicated than the alignment of coaxial systems. In addition, tolerances are also much more critical. These might be reasons why multi-tilted-mirror-telescopes are not so common. The preferred alignment follows the path of the wave fronts, which enter the final telescope (see e.g. Figure 4). In other words, the alignment starts at the entrance pupil of the system and ends at the detector plane. The first step is to provide a plane wave, which enters the preliminary telescope. The plane wave can be generated by an interferometer or by using e.g. an off-axis parabolic mirror, which collimates the light of a coherent point source. In this first state, the monocoque tube contains only the primary mirror M1. Thus, the place of the secondary mirror M2 is empty. The light will be focused by M1. Due to the fact that M1 is not an off-axis parabolic mirror, a defined aberration will be present, which is related to the tilt angle of M1. A wave front measurement can be carried out by using a Hartmann-Shack sensor ${ }^{5,6,7}$. And the correct position of M1 can be checked. A typical size of a Hartmann-Shack sensor is e.g. $15 \mathrm{~mm}$ x $15 \mathrm{~mm}$ or 
$36 \mathrm{~mm} \times 24 \mathrm{~mm}$ only. Thus, the wave front can be measured e.g. $3 \mathrm{~m}$ away from M1. The form measurement data of M1 can be implemented into the optical design software. The simulated optimal wave front can be compared with the results obtained by using the Hartmann-Shack sensor. After aligning M1 the secondary mirror M2 can be mounted. The interferometric form measurement data of M2, M3 and M4 also can be implemented into the optical design software. M2 provides a collimation. The derivative of the reasonable collimated wave front can be measured with a shearing plate ${ }^{6,8}$. This has to be done at least along two directions. The clear aperture of the shearing plate has to be $\varnothing \geq 200 \mathrm{~mm}$. This precision wedge type plane plate can be manufactured at the Technology Campus Teisnach, which is part of the DIT. The measurement can be carried out behind the mount of M3, which is not in place at this time. Once again, the measured wave front data are compared to the simulated data, which take the mirror measurement data into account. Thus, a wave front based alignment is realized. After aligning M2, M3 is mounted. The alignment of the plane mirror M3 is based on a wave position detection. This is done behind the M4 mount. M4 is detached at this moment. After the simple alignment of M3, M4 is mounted. Now, the Hartmann-Shack sensor will be used again in order to place M4 in its optimal position. Finally the z-position and the tilt of the detector plane has to be adjusted. This can be done by using the detected PSF. It is preferred to introduce a set of tilts between the entrance plane wave front and the telescope in order to compare the detected PSF over the entire FOV.

As a consequence of this alignment strategy, a closed CFRP monocoque tube can be used. In other words, it is not mandatory to implement open segments, which might be used in order to measure the local wave fronts. Thus, the CFRP monocoque tube, which can be seen at the right hand side of Figure 2, can be closed. This increases the mechanical stability of the telescope.

\section{CONCLUSION}

The students have great pleasure in the teamwork. The optics design, which is now scaled to $f / 8$ and thus somewhat relaxed, results in a Strehl ratio $\geq 0.8$ over the entire image field. However, a tolerance analysis has to be carried out (wave front deformations, installation faults in each with 6 degrees of freedom) in order to be able to estimate the practical feasibility.

It is intended to use of a Hartmann-Shack sensor and a shearing plate $(\varnothing \geq 200 \mathrm{~mm})$ for the alignment of the four tilted mirrors $5,6,7,8$. The adjustment takes place with the closed CFRP monocoque, i.e. successively starting from M1. The following mirrors are aligned one after another. The input wave front is provided by an interferometer (Fizeau, e.g. from Zygo) or a collimator (off-axis parabolic mirror, OAPM, preferably). The Hartmann-Shack sensor is used for the adjustment of M1 and M4. Here, this can be done because of the small size of the measured wave field. The alignment is carried out on the basis of the wave front measurement and by minimizing the deviation to the optimal condition, which should be available behind the individual mirrors M1 to M4. The shearing plate can be used behind M2 and M3 in order to determine the derivative of the wave font, which is present in shear direction. The measurement takes place at least along two orthogonal directions. It is intended to use the measurement data of the individual mirrors in Zemax. Thus, the real aberrations of the individual mirrors can be considered during the adjustment. The shearing plate can also be used to measure the input wave front. To do this the measuring aperture must be extended to $\varnothing=400 \mathrm{~mm}$ by stitching.

The mechanics and the electric drive of the parallactic mounting will cost approximately $40 \mathrm{k} €$. It is planned to finance the hardware costs mainly through sponsors. Interested parties are invited to participate in the non-commercial DIT student project. A rough estimate of the total costs - without personnel - is approximately $100 \mathrm{k} €$.

\section{PROOF OF AUTHORS CONTENT CONTRIBUTIONS}

GF: project management, initial optical design study, optical design selection and definition, parameter determination, setting f/7 system, definition of image performance. Definition of the practical field of view (FOV) and the detector parameter. Development of an alignment strategy, strategy for optical testing and definition of required optical components and sensors, as well as the implementation of measurement data, support SHK, BA, MA $(\notin \forall)$, no monetary benefit; AS: implementation and comparison of optical design (MA), AE: design tube and mirror mounts for mirrors M1-4 (SHK), SK: design fork mount (MA), MW: design gimbal mirror mount for primary mirror M1 in its first version, implementation of FEM and FEM simulation of the influence of the change of the position of M1 (BA); WK: tube design, exoskeleton, monocoque, carbon-fiber-reinforced-polymer (CFRP) plates and pipes (BA). 


\section{ACKNOWLEDGEMENT}

The author likes to thank the Faculty of Applied Natural Sciences and Industrial Engineering (NuW) of the Deggendorf Institute of Technology (DIT) for founding the related development project up to this point. This also includes fees for this paper, the conference and travel expenses. Thank you very much! Our thanks also goes to Prof. Dr. P. Sperber as main initiator, Prof. Dr. M. Moritz as Dean of the Faculty of Applied Natural Sciences and Industrial Engineering, as an optics design discussion partner and for the approval of student assistant $€$, E. Döberl and P. Keller from ASA for the good industry contact of the students, S. Sitzberger for the mechanics-support of students and explicitly to all students who have so far designed parts, e.g. within the general elective subject (GF-lecture: DIY Telescope making) or as student assistant. Thank all of you!

\section{REFERENCES}

[1] M. Brunn, DE3943258C2, „Silhouettierungsfreies Spiegelsystem für astronomische Teleskope vom Typ Schiefspiegler" (filed Dec. 29. 1989)

[2] M. Brunn, US5142417, „Unobscured All-Reflecting Telescopes Of The Schiefspiegler Type” (filed: Dec. 03. 1990).

[3] D. Stevick: „Stevick-Paul Telescopes”, http://www. amsky.com/atm/telescopes/spscopes/spt.html (online 06. 10. 2016).

[4] N. Morrison, "Introduction to Fourier analysis", John Wiley \& Sons Inc., New York (1994)

[5] J. Hartmann, „Bemerkungen über den Bau und die Justierung von Spektrographen“, Zeitschrift für Instrumentenkunde 20, September 1900, Seiten $17 \mathrm{ff}$ und $47 \mathrm{ff}$

[6] D. Malacara, "Optical Shop Testing", John Wiley \& Sons, 3rd edition, (2007).

[7] Naumann H., Schröder G., "Bauelemente der Optik", Carl Hanser Verlag, 6th Edition, (1992).

[8] M. Born, E. Wolf, "Principles of Optics", Pergamon Press (1991). 